\title{
Compliance with dietary guidelines affects capillary recruitment in healthy middle-aged men and women
}

\author{
Virginia Govoni ${ }^{1} \cdot$ Thomas A. B. Sanders $^{1} \cdot$ Dianne P. Reidlinger $^{1} \cdot$ Julia Darzi $^{1}$ \\ Sarah E. E. Berry ${ }^{1}$ Louise M. Goff ${ }^{1} \cdot$ Paul T. Seed $^{2} \cdot$ Philip J. Chowienczyk $^{3}$. \\ Wendy L. Hall ${ }^{1}$
}

Received: 14 August 2015 / Accepted: 23 December 2015 / Published online: 8 January 2016

(c) The Author(s) 2016. This article is published with open access at Springerlink.com

\begin{abstract}
Purpose Healthy microcirculation is important to maintain the health of tissues and organs, most notably the heart, kidney and retina. Single components of the diet such as salt, lipids and polyphenols may influence microcirculation, but the effects of dietary patterns that are consistent with current dietary guidelines are uncertain. It was hypothesized that compliance to UK dietary guidelines would have a favourable effect on skin capillary density/recruitment compared with a traditional British diet (control diet). Methods A 12-week randomized controlled trial in men and women aged 40-70 years was used to test whether skin microcirculation, measured by skin video-capillaroscopy on the dorsum of the finger, influenced functional capillary density (number of capillaries perfused under basal conditions), structural capillary density (number of anatomical capillaries perfused during finger cuff inflation) and capillary recruitment (percentage difference between structural and functional capillary density).

Results Microvascular measures were available for 137 subjects out of the 165 participants randomized to treatment. There was evidence of compliance to the dietary intervention, and participants randomized to follow dietary guidelines showed significant falls in resting supine
\end{abstract}

Wendy L. Hall

wendy.hall@kcl.ac.uk

1 Diabetes and Nutritional Sciences Division, Faculty of Life Sciences and Medicine, King's College London, 4.108 Franklin-Wilkins Building, 150 Stamford Street, London SE1 9NH, UK

2 Women's Health Division, King's College London, St Thomas' Hospital, London, UK

3 British Heart Foundation Centre, School of Medicine, King's College London, St Thomas' Hospital, London, UK systolic, diastolic and mean arterial pressure of 3.5, 2.6 and $2.9 \mathrm{mmHg}$ compared to the control diet. There was no evidence of differences in capillary density, but capillary recruitment was $3.5 \%(95 \% \mathrm{CI} 0.2,6.9)$ greater $(P=0.04)$ on dietary guidelines compared with control.

Conclusions Adherence to dietary guidelines may help maintain a healthy microcirculation in middle-aged men and women. This study is registered at www.isrctn.com as ISRCTN92382106.

Keywords Dietary guidelines - Cardiovascular disease . Microcirculation · Capillaroscopy $\cdot$ Blood pressure ·

Randomized controlled trial
Abbreviations
CVD Cardiovascular disease
BP Blood pressure
DG Dietary guidelines
CD Capillary density
CR Capillary recruitment

\section{Introduction}

Functional and structural modifications in the microcirculation occur early in the development of hypertension and diabetes [1-9], and it has been suggested that a reduction in the number of perfused microvessels at rest eventually leads to a loss of anatomical microvessels altogether (structural rarefaction) as well as functional impairment of capillary perfusion and recruitment $[1,2]$. Measuring microcirculation in the skin is recognized as a predictive, non-invasive marker of generalized microvascular and endothelial function [10], with studies showing impaired microvascular function to be concomitantly present in skin, 
coronary [11] and kidney [12] vessels. Skin microcirculation can be measured as capillary density, defined as the number of perfused vessels for unit area of tissue $\left(\mathrm{mm}^{2}\right)$, and can be distinguished as functional or structural. Functional density reflects the number of vessels perfused at rest, while structural density represents the total number of vessels present but only perfused when the metabolic demand of the tissue is greater [13], which is simulated by inflating a finger cuff to stop venous flow and measuring the density again after a few minutes. The difference between the two is called capillary recruitment (CR) and can be expressed as a percentage increase.

The restoration of microvascular function could help decrease systemic blood pressure (BP) by reducing peripheral vascular resistance, which in turn could improve circulation in target organs and help decrease cardiovascular disease (CVD) complications [14]. Evidence for the effect of diet on microcirculation is limited. The majority of published studies report the impact of single dietary components, such as salt [14, 15], omega-3 fatty acids [16] and polyphenols [17-19], in improving microvascular function. Current UK dietary guidelines (DG) for the prevention of CVD focus on maintaining a healthy weight, a reduction in the intakes of saturated fat, salt and added sugar, and increased consumption of fruit, vegetables, wholegrains and oily fish [20]. The aim of this study was to determine the effect of following a diet consistent with DG on skin capillary density/recruitment in men and women aged 40-70 years, compared with a traditional British dietary pattern.

\section{Participants and methods}

The CRESSIDA study (Cardiovascular disease risk REduction Study), registered at www.isrctn.com as 92382106, was a single-centre dietary intervention trial with a randomized, parallel design to test the effects of 12-week adherence to DG [21], compared with a traditional British dietary pattern $(\mathrm{CON})$, on $\mathrm{BP}$, vascular function and total cholesterol/HDL cholesterol ratio. The primary outcomes have been reported and showed significant reductions in daytime ambulatory systolic BP $[-4.2 \mathrm{mmHg}(95 \%$ CI $-6.6,-1.7 ; P<0.001)]$ and TC/HDL-C molar ratio $[-0.13(-0.26,0.00 ; P=0.044)]$ in the DG group compared to the CON group [22]. The study was conducted at King's College London between August 2010 and July 2012, approved by South London NHS Research Ethics Committee (Ref: 10/H0802/24), and received NHS R\&D approval from the Guy's and St Thomas' Hospitals Foundation Trust.

Detailed methodology for laboratory analysis, diet formulation and study design have been previously published [22].

\section{Participants}

Volunteers were recruited through newspaper advertisements and electronic internal circulars to staff and students at King's College London. All subjects gave written informed consent at the first screening visit, whereupon BP and anthropometric measurements were made, and urine and blood samples were collected for assessment of non-smoking status (urinary cotinine), and fasting blood lipid profile, glucose, liver function and haematology were assessed to confirm eligibility to take part in the study. The main inclusion criteria were: healthy and non-smoking men and women between the age of 40 and 70 years, while the exclusion criteria included a reported history of CVD, type 1 or type 2 diabetes mellitus, use of medication for lowering blood cholesterol or BP, body mass index (BMI) $<18.5$ and $>35 \mathrm{~kg} / \mathrm{m}^{2}$, an overall risk of CVD over the next 10 years $>20 \%$ estimated using the QRISK software (QRISK.org), clinical history of cancer (excluding basal cell carcinoma) in the past 5 years, chronic renal, liver or inflammatory bowel disease, history of substance abuse or alcoholism, current self-reported weekly alcohol intake exceeding $21 \mathrm{U}$ for women and $28 \mathrm{U}$ for men, pregnancy or having had a baby in the last 12 months. Furthermore, subjects were excluded if they reported being unwilling to follow the protocol and/or give informed consent, to refrain from use of dietary supplements, to restrict consumption of oily fish in accordance with the allocated diet or if they had a weight change of $>3 \mathrm{~kg}$ in the preceding 2 months.

\section{Study design}

Before randomization to treatment, eligible participants were asked to complete $2 \times 24$-h ambulatory BP measurements (results reported previously [22]) and urine collections, a 4-day food record, and validated food-frequency questionnaires. Treatment was allocated by minimization for age, sex, ethnicity and BMI by using a custom-designed computer database (MedSciNet AB). Participants attended the clinical research facility at St Thomas' Hospital, London, UK, in the fasting state for measurements of vascular function, including video-capillaroscopy, and to provide blood samples; procedures were repeated after 12 weeks. Full dietary advice based on the randomly allocated diet was given at baseline by a registered dietitian, and dietary assessments and lifestyle information were collected by questionnaires and 4-day food diary at baseline and at study endpoint.

\section{Dietary intervention}

The intervention diet (DG) was based on UK Department of Health dietary guidelines [21]. Targets for the DG group 
included reductions in the intake of total fat and saturated fatty acids to $<35$ and $<11 \%$ food energy, respectively, a reduction in added sugars to $<11 \%$ food energy, a reduction in salt intake to $<6 \mathrm{~g} /$ day, and an increase in fruit and vegetable, wholegrains and oily fish intake to $\geq 5, \geq 2$ portions/day and $\geq 1$ portions/week, respectively. The control diet (CON) was nutritionally balanced and based on a traditional British diet, formulated with familiar foods (full-fat milk, cheese, butter, meat and meat products, nonwholegrain cereals), and which reflected typical intakes of fruit and vegetables (3 portions/day), oily fish ( $<1$ portion/ month), saturated fatty acids ( $13 \%$ energy) and did not restrict intakes of salt or added sugar. Participants were counselled by a dietitian and provided with tailored advice. Both DG and CON groups were provided with some foods to aid compliance including pasta, microwavable rice, breakfast cereals, tinned beans and fish, nuts, cooking oils and spreads. Food intake records were checked and measurements of biomarkers of compliance were made to verify that the participants were following the advice, including measurements of 24-h urinary sodium, potassium, and sucrose plus fructose excretion as markers of salt, fruit and vegetables, and added sugars intake, respectively, and percentage changes in long chain n-3 PUFA in red blood cell membrane lipids (biomarker of oily fish intake) and plasma alkylresorcinols (biomarker of wholegrains intake).

\section{Measurement of skin capillary density and capillary recruitment}

Microvascular measures using skin video-capillaroscopy were secondary outcomes of the CRESSIDA study [22]. Measures were taken in accordance with standard operating procedures (SOPs) developed within the British Heart Foundation Centre at St Thomas' Hospital, based on published methodology and following best practice in vascular measures $[6,8,13,23]$. Capillary densities were measured on the dorsum of the middle phalanx of the third finger of the left hand, or fourth finger if rings were present and not removable, using the CAM1 Capillary Anemometer and CapiScope ${ }^{\mathrm{TM}}$ System (KK Research Technology Ltd., Devon, UK) in a temperature-controlled room (mean $22 \pm 1.8^{\circ} \mathrm{C}$ ). Analyses were carried out in the morning in a fasted state; prior to this, participants consumed standardized low-fat/low-salt meals the evening before. After 30-min supine rest, measurements were made of supine $\mathrm{BP}$ in triplicate at 5-min intervals using the Omron $705 \mathrm{CP}$ device. Following this, measurements of carotid-femoral pulse wave velocity, radial pulse wave analysis and digital volume pulse wave were made, and after a further $20 \mathrm{~min}$, capillary measurements were made. The left arm was supported at heart level in a comfortable position with the finger positioned under the microscope and kept in place with a plastic finger clamp to limit movement. The site of skin to be analysed was covered with drops of vegetable oil to limit light reflection. Measurements were taken at baseline (before dietary intervention at week 0 ) and following the 12-week dietary intervention.

Four fields were identified around a central point and recorded for $10 \mathrm{~s}$ each before and after venous occlusion. This was achieved by inflating a small finger cuff positioned at the base of the finger to $60 \mathrm{mmHg}$ for $5 \mathrm{~min}$. Total numbers of capillaries in each video, visible as black dots on a grey background, were counted offline using the CapiScope $^{\mathrm{TM}}$ automatic software and manually corrected for movement artefacts. Functional capillary density (FCD) was calculated from the average density of the four fields taken at rest, and structural capillary density (SCD) as the average density taken after 5 min of cuff inflation. Densities are reported as number of vessels per $\mathrm{mm}^{2}$.

Capillary recruitment was derived by subtracting FCD values from the SCD and expressing it as a percentage. Reproducibility was tested in ten healthy subjects before starting the intervention. The day-to-day reproducibility of the technique was $4.2 \%$ for FCD and $3.2 \%$ for structural CD with correlation coefficients of $0.9(P=0.001)$ for both measures. Within-day intra-observer reproducibility was also acceptable, with a CV of $7.1 \%$ for FCD and $5.9 \%$ for SCD.

\section{Statistical analysis}

Analyses were performed with Stata version 13.1 (Stata Corp). All variables were checked for normality. Variables that were not normally distributed were natural log-transformed, and the data expressed as geometric mean. Data were analysed on a per protocol basis by analysis of covariance with the endpoint value regressed against the baseline value, gender, age and BMI categories; the treatment effect is the regression-adjusted difference between groups. Causal mediated effect analysis, which estimates the extent to which the specified effect of the randomized treatment is mediated through its other effects, was conducted to explore whether the changes in CR could be explained by changes in sodium excretion, BMI and systolic BP [24].

\section{Results}

Out of 165 participants randomized to treatment, 162 participants completed the study and data were available for analysis in 137 participants (72 DG, $65 \mathrm{CON}$ ). Data were missing for 15 participants because the technique could not be performed on darkly pigmented skin and on ten participants due to missing measurements. Figure 1 shows the flow of participants through the study, and Table 1 shows their characteristics. 
Fig. 1 Consort diagram
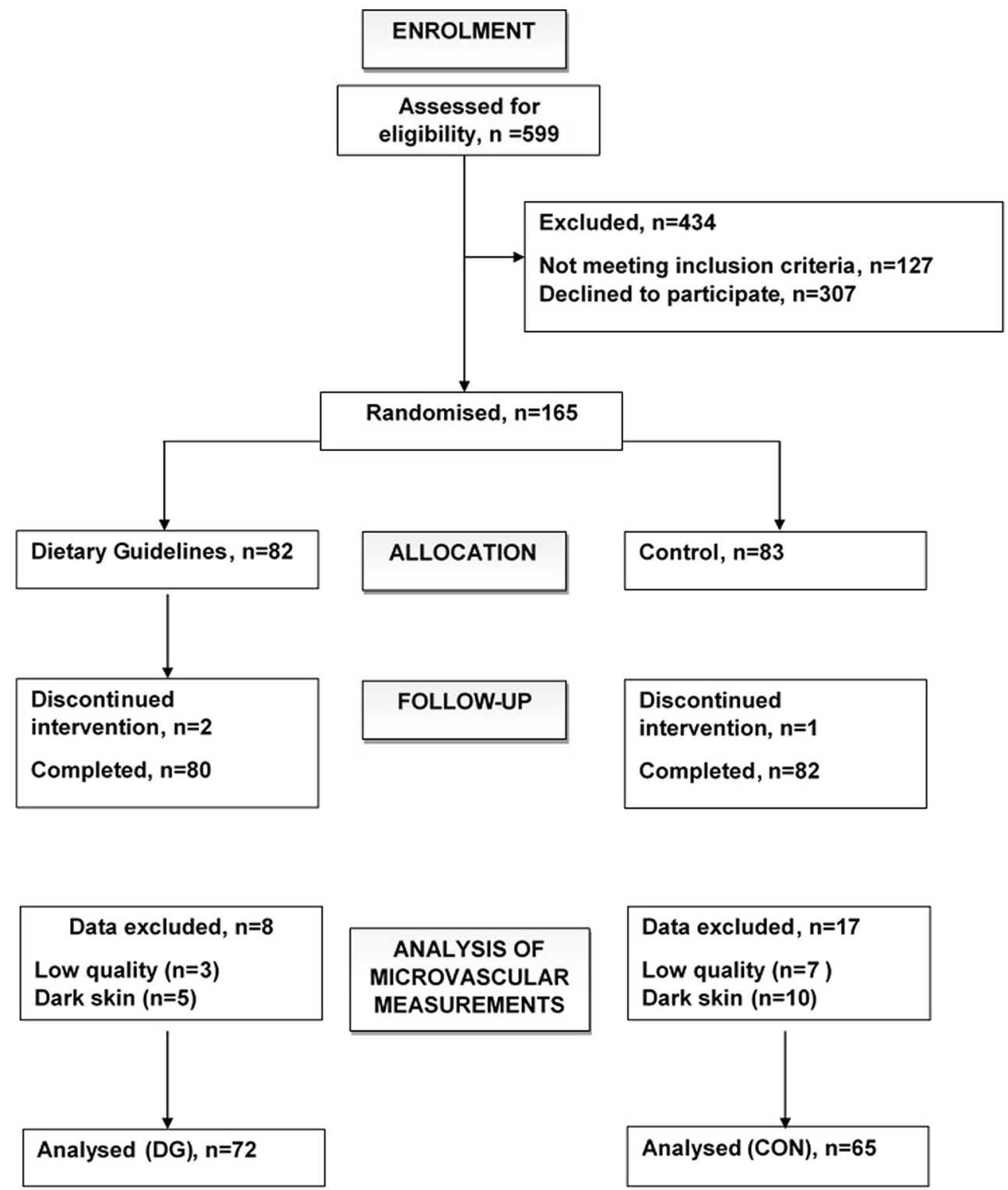

\section{ANALYSIS OF MICROVASCULAR MEASUREMENTS}

\section{Data excluded, $n=17$ \\ Low quality $(n=7)$}

Dark skin $(n=10)$

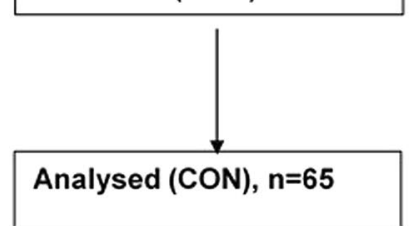

The participants were on average aged 53 years old with a BMI of $26 \mathrm{~kg} / \mathrm{m}^{2}$. They were predominantly normotensive (seated BP $<140 / 90 \mathrm{mmHg}$ ) and of white European origin. Non-smoking status was confirmed by urinary cotinine analysis. Over half of the women were post-menopausal. Table 2 reports the main dietary goals and the biomarkers used to check compliance to the diets. There was no evidence of differences between groups in their baseline dietary intakes or baseline characteristics. Compliance to dietary advice on both DG and CON diets was verified by reported dietary intakes and analysis of biomarkers. Saturated fatty acid intakes were reduced to target levels in $95 \%$ of the DG group, and this was accompanied by a $10 \%$ fall in low-density lipoprotein (LDL) cholesterol as would be expected following reduced saturated fatty acid intakes. Decreases in urinary sucrose and fructose excretion in the DG group indicated compliance to advice to reduce intake of added sugars. An increase in wholegrain consumption on DG was evidenced by increased plasma alkylresorcinol concentrations. Compliance to oily fish advice was demonstrated by increases in the proportions of eicosapentaenoic (20:5n-3) and docosahexaenoic (22:6n3) acid in erythrocyte lipids. Reported fruit and vegetable intakes were already close to target at baseline, an observation that was supported by urinary potassium excretion which was generally high. Although reported fruit and vegetable servings per day were increased in the DG group compared to $\mathrm{CON}$, there was no significant difference between groups in terms of potassium excretion, an indication that all participants were consuming plenty of fruit and vegetables. In addition to lower dietary sodium intakes estimated from 4-day food diaries, urinary sodium excretion was markedly reduced by the DG intervention. 
Table 1 Details of male and female participants by randomized treatment group

\begin{tabular}{lll}
\hline & $\begin{array}{l}\text { DG } \\
n=72\end{array}$ & $\begin{array}{l}\text { CON } \\
n=65\end{array}$ \\
\hline Age & $53 \pm 8$ & $53 \pm 8$ \\
Sex & & \\
Male, $n(\%)$ & $31(43)$ & $26(40)$ \\
Pre-menopausal female, $n(\%)$ & $15(21)$ & $15(23)$ \\
Post-menopausal female, $n(\%)$ & $26(36)$ & $24(37)$ \\
Ethnicity & & \\
White European, $n(\%)$ & $66(92)$ & $61(94)$ \\
Asian, $n(\%)$ & $6(8)$ & $4(6)$ \\
BMI (kg/m $\left.{ }^{2}\right)$ & $25.0 \pm 3.3$ & $26.5 \pm 3.7$ \\
Fasting plasma total cholesterol (mmol/L) & $5.3 \pm 1.0$ & $5.3 \pm 0.9$ \\
Fasting plasma HDL cholesterol (mmol/L) & $1.6 \pm 0.4$ & $1.5 \pm 0.5$ \\
Fasting plasma TG (mmol/L) & $1.0 \pm 0.6$ & $1.2 \pm 0.5$ \\
Fasting plasma glucose (mmol/L) & $5.3 \pm 0.5$ & $5.2 \pm 0.4$ \\
Seated systolic BP (mmHg) & $118 \pm 14$ & $119 \pm 13$ \\
Seated diastolic BP (mmHg) & $76 \pm 8$ & $78 \pm 9$ \\
10-year risk of CVD men (\%) & $7.6 \pm 5.3$ & $7.9 \pm 5.7$ \\
10-year risk of CVD women (\%) & $3.7 \pm 3.1$ & $4.9 \pm 3.4$ \\
\hline
\end{tabular}

Mean \pm SD. 10-year risk of CVD estimated using QRISK-2. No significant differences between groups: two-sample $t$ test or MannWhitney $U$ test

$D G$ dietary guidelines, $C O N$ control, $B M I$ body mass index, $B P$ blood pressure, $C V D$ cardiovascular disease, $H D L$ high-density lipoprotein

\section{Effect on blood pressure and microcirculation}

Supine peripheral systolic, diastolic and mean arterial BP all fell significantly (all $P<0.001$ ) on the DG compared with $\mathrm{CON}$ by $3.4,2.5$ and $2.9 \mathrm{mmHg}$, respectively (Table 3). There were no significant differences in functional or structural capillary density at baseline, but following the intervention, CR was $3.5 \%$ greater following DG compared with CON which was statistically significant $(P=0.04)$. This significant difference remained after adjusting for changes in mean arterial pressure and baseline sodium excretion. Causal mediated effect analyses did not find significant effects for changes in urinary sodium excretion or changes in weight.

\section{Discussion}

Adherence to DG increased post-occlusive CR in the skin relative to CON, suggesting improved regulation of blood flow in the microcirculation. The differences between treatment groups were marginal, and definitive conclusions may only be drawn once further studies have confirmed these effects. Improved capillary function may have been an indirect consequence of reduced peripheral resistance which increases tissue perfusion. BP-lowering drugs (ACE inhibitors, angiotensin-II receptor antagonist and some diuretics)
Table 2 Comparison of dietary intakes estimated from 4-day food diaries at baseline and following intervention, together with biomarkers of compliance on treatment, compared with targets for the dietary guidelines group

\begin{tabular}{lllll}
\hline & Target $^{\$}$ & $\begin{array}{l}\text { Baseline } \\
n=137\end{array}$ & $\begin{array}{l}\text { DG } \\
n=72\end{array}$ & $\begin{array}{l}\text { CON } \\
n=65\end{array}$ \\
\hline Total fat (\% food energy) & & & & \\
SFA (\% food energy) & $<5$ & $35.7 \pm 5.9$ & $34.0 \pm 5.3^{*}$ & $37.7 \pm 5.3$ \\
LDL-cholesterol (mmol/L) & & $12.1 \pm 3.3$ & $7.7 \pm 1.8^{*}$ & $15.0 \pm 2.8$ \\
NMES (\% food energy) & $<11$ & $9.7 \pm 4.4$ & $7.1 \pm 4.0^{*}$ & $9.4 \pm 3.8$ \\
24-h urinary sucrose and fructose ( $\mu$ mol/24 h) & & $41(33,50)$ & $28(21,38)^{*}$ & $56(43,73)$ \\
Wholegrains (servings/day) & $\geq 2$ & $1.4 \pm 1.1$ & $2.7 \pm 1.36^{*}$ & $1.0 \pm 1$ \\
Plasma alkylresorcinol (nmol/L) & & $75(66,85)$ & $87(74,102)^{*}$ & $64(53,78)$ \\
Oily fish (servings/week) & $\geq 1$ & $1.0 \pm 1.0$ & $1.7 \pm 1.56^{*}$ & $0.2 \pm 0.3$ \\
Erythrocyte 20:5n-3 + 22:6n-3 (wt\%) & & $7.1 \pm 2.2$ & $8.0 \pm 2.4^{*}$ & $6.7 \pm 2.1$ \\
Fruit and vegetables (servings/day) & $\geq 5$ & $5 \pm 3$ & $7 \pm 3 *$ & $5 \pm 3$ \\
24-h urinary potassium (mmol/day) & & $83 \pm 29$ & $86 \pm 30$ & $81 \pm 22$ \\
Sodium intake (mmol/day) & $<100$ & $142 \pm 53$ & $84 \pm 83^{*}$ & $152 \pm 59$ \\
24-h urinary Na excretion (mmol/day) & & $125 \pm 55$ & $80 \pm 39^{*}$ & $133 \pm 55$ \\
\hline
\end{tabular}

Mean values $\pm \mathrm{SD}$ or geometric mean $(95 \% \mathrm{CI})$

There were no statistical differences between DG and CON at baseline

$D G$ dietary guidelines, NMES non-milk extrinsic sugars

* Significant difference from control $P<0.01$; two-sample $t$ test

$\$ 140 \mathrm{~g}$ oily fish $=1$ serving; $30 \mathrm{~g}$ wholegrains $=1$ serving; $80 \mathrm{~g}$ fruit or vegetable $=1$ serving 
Table 3 Changes in supine blood pressure (BP) and digital microcirculation in healthy non-smoking men and women aged $40-70$ years before and 12 weeks after random allocation to dietary guidelines (DG) or control (CON) diets

\begin{tabular}{|c|c|c|c|c|}
\hline & $\begin{array}{l}\text { DG } \\
(n=72)\end{array}$ & $\begin{array}{l}\mathrm{CON} \\
(n=65)\end{array}$ & Treatment effect & $\begin{array}{l}P \\
\text { value }\end{array}$ \\
\hline \multicolumn{5}{|c|}{ Supine systolic BP $(\mathrm{mmHg})$} \\
\hline Baseline & $117(114,120)$ & $116(113,119)$ & & \\
\hline Endpoint & $112(110,115)$ & $115(113,118)$ & $-3.5(-5.4,-1.7)$ & $<0.001$ \\
\hline \multicolumn{5}{|c|}{ Supine diastolic $B P(\mathrm{mmHg})$} \\
\hline Baseline & $74(72,76)$ & $74(72,76)$ & & \\
\hline Endpoint & $71(69,73)$ & $74(72,76)$ & $-2.6(-3.8,-1.4)$ & $<0.001$ \\
\hline \multicolumn{5}{|c|}{ Supine mean arterial BP $(\mathrm{mmHg})$} \\
\hline Baseline & $88(86,90)$ & $88(86,90)$ & & \\
\hline Endpoint & $85(83,87)$ & $88(86,90)$ & $-2.9(-4.2,-1.6)$ & $<0.001$ \\
\hline \multicolumn{5}{|c|}{ Functional capillary density $\left(\mathrm{n} / \mathrm{mm}^{2}\right)$} \\
\hline Baseline & $110(106,113)$ & $110(105,114)$ & & \\
\hline Endpoint & $108(104,112)$ & $102(102,110)$ & $1.1(-2.8,5.1)$ & 0.57 \\
\hline \multicolumn{5}{|c|}{ Structural capillary density $\left(\mathrm{n} / \mathrm{mm}^{2}\right)$} \\
\hline Baseline & $116(111,120)$ & $113(109,118)$ & & \\
\hline Endpoint & $118(113,122)$ & $113(109,116)$ & $2.5(-0.8,5.9)$ & 0.14 \\
\hline \multicolumn{5}{|c|}{ Capillary recruitment (\% increase) } \\
\hline Baseline & $6.4(4.3,8.4)$ & $3.7(1.9,5.6)$ & & \\
\hline Endpoint & $9.7(7.3,12.1)$ & $6.5(4.3,8.6)$ & $3.5(0.2,6.9)$ & 0.04 \\
\hline
\end{tabular}

Mean values $(95 \% \mathrm{CI})$. Data were analysed on a per protocol basis, and the probabilities are based on analysis of covariance with value on treatment regressed against the baseline value, gender, age and BMI categories; the treatment effect is the regression-adjusted difference between groups improve microvascular function in both animals [25] and humans [26], with mechanisms including activation of bradykinin pathways, stimulation of vascular endothelial growth factor production and increased nitric oxide formation. However, as previously reported, we were unable to demonstrate any effect of diet on nitric oxide-dependent vasorelaxation of the brachial artery [22], nor measures of radial pulse wave augmentation index or digital volume pulse reflection index (unpublished data). The improvement in skin CR following the DG diet compared to a traditional British diet appeared to be independent of the changes in BP.

He et al. [14] reported skin capillary microcirculation data from a crossover trial of slow sodium (sodium chloride tablets in slow release form) compared to placebo, with each intervention lasting 6 weeks in 169 hypertensive subjects following a reduced salt diet (mean BP $147 / 91 \mathrm{mmHg}$ ). Salt intake on slow sodium was $9.7 \mathrm{~g} /$ day (159 $\mathrm{mmol} / \mathrm{day})$ compared to $6.5 \mathrm{~g} /$ day $(111 \mathrm{mmol} / \mathrm{day})$ on placebo. FCD was significantly lower following slow sodium treatment compared to placebo (101 and 106 capillaries per $\mathrm{mm}^{2}$, respectively), and the same was observed for SCD following 2-min venous congestion (108 and 115 capillaries per $\mathrm{mm}^{2}$, respectively). In the present study, the duration of intervention was longer, but CRESSIDA participants were non-smokers and mainly normotensive. These factors may explain the discrepancy with the findings of
He and colleagues as regards the lack of effect observed on resting capillary density with an integrated dietary intervention that included salt restriction. The capillary densities in our participants were similar to the values reported by $\mathrm{He}$ et al. in hypertensives following sodium restriction (106 capillaries per $\mathrm{mm}^{2} \mathrm{FCD}$ and 115 capillaries per $\mathrm{mm}^{2}$ SCD) and are similar to values reported in other studies in predominantly normotensive individuals $[8,27-30]$. Furthermore, it should be noted that the microvascular measures were a secondary outcome of the current study, and the possibility that the study was underpowered to detect changes in capillary density cannot be excluded.

A significant novelty of the study is that the dietary intervention comprised a whole diet approach. Evidence in healthy populations showing that adherence to combined DG might modify markers of risk of CVD is scarce. Most studies focus on single components of dietary advice and are often conducted in high-risk populations or populations with CVD. The present study was conducted in nonsmoking individuals at average risk of CVD who were not receiving medication for lipids and BP. The study included a relatively large sample size and a relevant duration for observing changes in vascular function. Furthermore, good compliance to the dietary intervention was achieved as evidenced by validated biomarkers of dietary compliance and the falls in BP. Other than a slightly higher intake of saturated fat (by $8 \mathrm{~g} /$ day), the CON diet was nutritionally 
balanced and was not markedly different from the participants' usual diet. The study indicates the expected size of impact on microvascular function to be expected in a healthy middle-aged population adhering to DG. It was not possible to attribute through causal mediated effect analysis the components of the diet that were responsible. This may have been due to a lack of statistical power. Alternatively, the effect may be the sum of several components of the intervention.

An important limitation of the capillaroscopy method was that we were unable to make measurements in participants with heavily melanized skin. He et al., however, reported no ethnic differences in response to salt load using orthogonal polarization spectral imaging at the side of the fingers in participants of black African origin. Nevertheless, the findings reported here need to be confirmed in additional studies using techniques that are not restricted to populations with lightly melanized skin, for example orthogonal polarization spectroscopy (OPS). The numerical values of capillary densities in this cohort of healthy individuals with normal to low BP are in accordance with the published literature [8, 27-30], although meaningful comparisons with other studies are difficult due to differing techniques, BP status and smoking status. Furthermore, the data reinforce the limited information available on ranges of FCD, SCD and CR available in the literature, especially in healthy men and women older than 40 years. Changes in response to diet may differ according to sex, age and ethnic background; future studies should be designed and statistically powered to consider the influence of these inter-individual factors.

Microcirculation could be an important target to help prevent CVD or limit the progression of vascular changes, which happen very early in the development of metabolic conditions. Our findings show that adherence to DG imparted some benefit for microvascular function in a healthy population of middle-aged to older men and women, despite being a predominantly normotensive population.

Acknowledgments We acknowledge Zoe Maniou, Sarah Cottin, Ann Donald, Angela Shore, Karen McNeil, Robert Gray, Tracy Dew and Laura O'Sullivan for their contribution to data collection and analysis. The research was supported by funding from the UK Food Standards Agency and Department of Health and also supported by the National Institute for Health Research (NIHR) Clinical Research Facility at Guy's \& St Thomas' NHS Foundation Trust and NIHR Biomedical Research Centre based at Guy's and St Thomas' NHS Foundation Trust and King's College London. The research was also supported by Tommy's (registered charity no. 1060508) \& National Institute for Health Research (NIHR) Collaboration for Leadership in Applied Health Research and Care South London at King's College Hospital NHS Foundation Trust. The views expressed are those of the author(s) and not necessarily those of Tommy's, the NHS, the NIHR or the Department of Health. Some foods for the study were supplied by Archer Daniel Mills PLC, Veetee Rice, Heinz PLC, Almond Board of California and the Southern African Macadamia Growers Association. Donor companies were not involved in the trial design, data collection, data analysis or reporting of the results.

Author contribution TABS was the principal investigator and guarantor of the study. TABS, WLH, PJC, LG, PS, SEB and JD designed the research. VG conducted the measurements of capillary function, and VG, TABS and PS carried out data analysis. JD and DPR recruited subjects onto the study. DPR supported the dietary intervention and analysed dietary data. JD oversaw the organization of the trial and analysis of biomarkers of dietary compliance. VG, WLH and TABS wrote the paper. WLH has primary responsibility for the final content of the manuscript. All authors read and approved the final manuscript.

\section{Compliance with ethical standards}

Conflict of interest Thomas Sanders is a trustee and governor of the British Nutrition Foundation and reported a financial interest in respect of payment for attendance at scientific advisory panels for Heinz PLC, Global Dairy Platform, Malaysian Palm Oil Board and the Natural Hydration Council, and GlaxoSmithKline and lecture fees from Lilly. PJC reported a financial interest in Centron Diagnostics. DPR, JD, LG, WLH, PTS, SEB and VG reported no conflicts of interest.

Open Access This article is distributed under the terms of the Creative Commons Attribution 4.0 International License (http://creativecommons.org/licenses/by/4.0/), which permits unrestricted use, distribution, and reproduction in any medium, provided you give appropriate credit to the original author(s) and the source, provide a link to the Creative Commons license, and indicate if changes were made.

\section{References}

1. Feihl F, Liaudet L, Waeber B (2009) The macrocirculation and microcirculation of hypertension. Curr Hypertens Rep 11:182-189

2. Serne EH, Gans RO, ter Maaten JC, ter Wee PM, Donker AJ, Stehouwer CD (2001) Capillary recruitment is impaired in essential hypertension and relates to insulin's metabolic and vascular actions. Cardiovasc Res 49:161-168

3. Jorneskog G, Brismar K, Fagrell B (1995) Skin capillary circulation severely impaired in toes of patients with IDDM, with and without late diabetic complications. Diabetologia 38:474-480

4. Jorneskog G, Fagrell B (1996) Discrepancy in skin capillary circulation between fingers and toes in patients with type 1 diabetes. Int J Microcirc Clin Exp 16:313-319

5. Barchetta I, Riccieri V, Vasile M, Stefanantoni K, Comberiati P, Taverniti L, Cavallo MG (2011) High prevalence of capillary abnormalities in patients with diabetes and association with retinopathy. Diabet Med 28:1039-1044

6. Tibirica E, Rodrigues E, Cobas R, Gomes MB (2007) Impairment of skin capillary recruitment precedes chronic complications in patients with type 1 diabetes. Rev Diabet Stud 4:85-88

7. Jaap AJ, Hammersley MS, Shore AC, Tooke JE (1994) Reduced microvascular hyperaemia in subjects at risk of developing type 2 (non-insulin-dependent) diabetes mellitus. Diabetologia 37:214-216

8. Antonios TF, Rattray FM, Singer DR, Markandu ND, Mortimer PS, MacGregor GA (2003) Rarefaction of skin capillaries in normotensive offspring of individuals with essential hypertension. Heart 89:175-178

9. Noon JP, Walker BR, Webb DJ, Shore AC, Holton DW, Edwards HV, Watt GC (1997) Impaired microvascular dilatation and 
capillary rarefaction in young adults with a predisposition to high blood pressure. J Clin Invest 99:1873-1879

10. Holowatz LA, Thompson-Torgerson CS, Kenney WL (2008) The human cutaneous circulation as a model of generalized microvascular function. J Appl Physiol 105:370-372

11. Ijzerman R, de Jongh RT, Beijk MA, van Weissenbruch MM, Delemarre-van de Waal HA, Serne EH, Stehouwer CD (2003) Individuals at increased coronary heart disease risk are characterized by an impaired microvascular function in skin. Eur J Clin Invest 33:536-542

12. Kruger A, Stewart J, Sahityani R, O'Riordan E, Thompson C, Adler S, Garrick R, Vallance P, Goligorsky MS (2006) Laser Doppler flowmetry detection of endothelial dysfunction in endstage renal disease patients: correlation with cardiovascular risk. Kidney Int 70:157-164

13. Shore AC (2000) Capillaroscopy and the measurement of capillary pressure. Br J Clin Pharmacol 50:501-513

14. He FJ, Marciniak M, Markandu ND, Antonios TF, MacGregor GA (2010) Effect of modest salt reduction on skin capillary rarefaction in white, black, and Asian individuals with mild hypertension. Hypertension 56:253-259

15. de Jongh RT, Serne EH, IJzerman RG, Stehouwer CD (2007) Microvascular function: a potential link between salt sensitivity, insulin resistance and hypertension. J Hypertens 25:1887-1893

16. Mori TA, Watts GF, Burke V, Hilme E, Puddey IB, Beilin LJ (2000) Differential effects of eicosapentaenoic acid and docosahexaenoic acid on vascular reactivity of the forearm microcirculation in hyperlipidemic, overweight men. Circulation 102:1264-1269

17. Neukam K, Stahl W, Tronnier H, Sies H, Heinrich U (2007) Consumption of flavanol-rich cocoa acutely increases microcirculation in human skin. Eur J Nutr 46:53-56

18. Heinrich U, Moore CE, De Spirt S, Tronnier H, Stahl W (2011) Green tea polyphenols provide photoprotection, increase microcirculation, and modulate skin properties of women. J Nutr 141:1202-1208

19. Goncalves MC, Bezerra FF, Eleutherio EC, Bouskela E, Koury J (2011) Organic grape juice intake improves functional capillary density and postocclusive reactive hyperemia in triathletes. Clinics (Sao Paulo) 66:1537-1541

20. Department of Health (1994) Nutritional Aspects of Cardiovascular Disease. Report on Health and Social Subjects No 46. HMSO. London
21. SACN (2008) Scientific Advisory Committee on Nutrition. The nutritional wellbeing of the British population (Internet). London: Scientific Advisory Committee on Nutrition. 2008 (cited 6 Aug 2014). http://www.sacn.gov.uk/pdfs/nutritional_health_of_ the_population_final_oct_08.pdf

22. Reidlinger DP, Darzi J, Hall WL, Seed PT, Chowienczyk PJ, Sanders TAB, Cardiovasc Dis Risk R (2015) How effective are current dietary guidelines for cardiovascular disease prevention in healthy middle-aged and older men and women? a randomized controlled trial. Am J Clin Nutr 101:922-930

23. Antonios TF, Rattray FE, Singer DR, Markandu ND, Mortimer PS, MacGregor GA (1999) Maximization of skin capillaries during intravital video-microscopy in essential hypertension: comparison between venous congestion, reactive hyperaemia and core heat load tests. Clin Sci (Lond) 97:523-528

24. Imai K, Keele L, Yamamoto T (2010) Identification, inference and sensitivity analysis for causal mediation effects. Stat Sci 25:51-71

25. Levy BI, Duriez M, Samuel JL (2001) Coronary microvasculature alteration in hypertensive rats: effect of treatment with a diuretic and an ACE inhibitor. Am J Hypertens 14:7-13

26. Olsen MH, Wachtell K, Neland K, Bella JN, Rokkedal J, DigePetersen H, Ibsen H (2005) Losartan but not atenolol reduce carotid artery hypertrophy in essential hypertension: a LIFE substudy. Blood Press 14:177-183

27. Karatzi K, Protogerou A, Kesse-Guyot E, Fezeu LK, Carette C, Blacher J, Levy BI, Galan P, Hercberg S, Czernichow S (2014) Associations between dietary patterns and skin microcirculation in healthy subjects. Arterioscler Thromb Vasc Biol 34:463-469

28. Debbabi H, Uzan L, Mourad JJ, Safar M, Levy BI, Tibirica E (2006) Increased skin capillary density in treated essential hypertensive patients. Am J Hypertens 19:477-483

29. Antonios TF, Singer DR, Markandu ND, Mortimer PS, MacGregor GA (1999) Structural skin capillary rarefaction in essential hypertension. Hypertension 33:998-1001

30. Levy BI, Schiffrin EL, Mourad JJ, Agostini D, Vicaut E, Safar ME, Struijker-Boudier HA (2008) Impaired tissue perfusion: a pathology common to hypertension, obesity, and diabetes mellitus. Circulation 118:968-976 